\title{
CONTROL OF TRIACYLGLYCEROL BIOSYNTHESIS IN PLANTS
}

Recent publications which have resulted at least in part from DOE support are listed below:

1. Jaworski, J.G., Post-Beittenmiller, D. and Ohlrogge, J.B. 1993 Acetyl-ACP is not a major intermediate in fatty acid biosynthesis in plants. Eur. J. Biochem. in press

2. Post-Beittenmiller, D., Roughan, P.G., and Ohlrogge, J.B. 1992 Regulation of plant fatty acid biosynthesis: Analysis of acyl-CoA and acyl-ACP substrate pools in spinach and pea chloroplasts. Plant Physiol. 100, 923-930.

3. Dörmann, P., Spener, F., and Ohlrogge, J.B. 1993 Characterization of two acyl-ACP thioesterases specific for medium chain acyl-ACP and oleoyl-ACP from developing Cuphea seeds. Planta in press

4. Post-Beittenmiller, D., Ohlrogge, J.B., and Somerville, C.R. 1993 Regulation of plant lipid biosynthesis: An example of developmental regulation superimposed on a ubiquitous pathway. In: Control of Plant Gene Expression. D.P.S. Verma ed. in press.

5. Hloušek-Radojčić, A., Post-Beittenmiller, D., and Ohlrogge, J.B. 1992 Expression of constitutive and tissue-specific acyl carrier protein isoforms in Arabidopsis. Plant Physiology, 98, 206-214.

6. Cahoon, E.B., Shanklin, J. and Ohlrogge, J.B. 1992 Expression of a novel coriander desaturase results in petroselinic acid production in transgenic tobacco. Proc.Natl.Acad.Sci. 89, 11184-11188.

\section{SUMMARY OF MOST RECENT WORK}

Apparent Role of Phosphatidylcholine in the Metabolism of Petroselinic Acid in Developing Umbelliferae Endosperm

Seeds of most species of the Umbelliferae (Apiaciae), Araliaceae, and Garryaceae families are characterized by their high content of the unusual $C_{18}$ monounsaturated fatty acid petroselinic acid (18:1 $\Delta^{6 c i n}$. Prior to a recent report of this lab (publication \#6, above), little was known of the biosynthetic origin of the cis $\Delta^{6}$ double bond of petroselinic acid. Such knowledge may be of both biochemical and biotechnological significance. Because petroselinic acid is potentially the product of a novel desaturase, information regarding its synthesis may contribute to an understanding of fatty acid desaturation mechanisms in plants. In addition, petroselinic acid possesses possible industrial value. Through chemical cleavage at its double bond, petroselinic acid can be used as a precursor of lauric acid (12:0), a component of detergents and surfactants, and adipic acid (6:0 dicarboxylic), the monomeric component of nylon 6,6. Therefore, the development of an agronomic source of an oil rich in netroselinic acid is of biotechnological interest (Murphy, 1992). As such, studies of petroselinic acid biosynthesis may provide basic information required for any attempt to genetically engineer the production and accumulation of this fatty acid in an existing oilseed.

Because of its structural similarity to oleic acid, petroselinic acid offers an interesting case study of the metabolism of an unusual fatty acid in a developing oilseed. Unusual fatty acids of seeds, especially acyl groups of atypical chemical structures, are often absent or found in only small amounts in membrane (or 
polar) glycerolipids including phosphatidylcholine (PC), the primary membrane lipid of seeds. These fatty acids are instead concentrated in a storage form as triacylglycerol (TAG) (as reviewed by Ohlrogge, 1988; Battey et al., 1989). In theory, the partitioning of unusual fatty acids away from polar glycerolipids insures that "proper" membrane function is not disrupted by the aberrant structures of these acyl moieties (Stymne et al., 1991). It has been proposed that this selective partitioning of unusual fatty acids may result from the substrate specificities of enzymes such as diacylglycerol acyltransferase and diacylglycerol:CDP-choline phosphotransferase (Bafor et al., 1990; Browse and Somerville, 1991). Together these two enzymes serve as primary determinants of fatty acid flux via diacylglycerol into storage (TAG) and membrane glycerolipids. It has also been suggested that specialized acyltransferases may contribute to the channeling of unusual fatty acids into TAG (Oo and Huang, 1989). An example of such an enzyme is a lysophosphosphatidic acid acyltransferase characterized in extracts of palm endosperm which displays a marked preference for mediumchain length fatty acids (Oo and Huang, 1989).

In contrast, oleic acid and its polyunsaturated derivatives (e.g., $\alpha$-linoleic acid and $\alpha$-linolenic acid) are not excluded from PC in developing oilseeds such as those of soybean, safflower, and linseed (Slack $e t$ al., 1978). In these tissues, $\mathrm{PC}$ appears to readily participate in the flux of $\mathrm{C}_{18}$ polyunsaturated fatty acids into TAG. In addition, the unusual fatty acids $\gamma$-linolenic acid and ricinoleic acid may also be metabolized through PC in seeds of borage (Stymne and Stobart, 1986; Stymne and Stobart, 1988) and castor (Bafor $e t$ al., 1991), repectively. In each of these cases, however, the metabolic flow of fatty acids through PC can be attributed to the role of this lipid as a substrate for fatty acid modification reactions including desaturation and hydroxylation.

We are currently investigating the metabolism of the unusual fatty acid petroselinic acid in the endosperm of developing seeds of the Umbelliferae species coriander and carrot. As reported below, petroselinic acid accumulates to high levels in TAG but to significantly lower levels in the major membrane glycerolipids e.g., PC and phosphatidylethanolamine (PE). However, a high flux of petroselinic acid appears to be maintained through both stereospecific positions of $\mathrm{PC}$ as judged by $\left[1-{ }^{14} \mathrm{C}\right]$ acetate labelling. The apparent movement of petroselinic acid through PC (and perhaps other polar lipids) occurs despite the fact that petroselinic acid is neither synthesized nor further modified on PC in coriander or carrot endosperm (Cahoon et al., 1992; Cahoon and Ohlrogge, in prep.).

\section{Distribution of Petroselinic Acid in Glycerolipids of Carrot and Coriander Endosperm}

Petroselinic acid has been previously identified in phosphatidylcholine (Dutta et al., 1992) and the total polar lipid fraction of carrot seed (Dutta and Appelqvist, 1991) as well as the total phospholipids of carrot and coriander seed (Prasad et al., 1987). A more detailed analysis indicated that petroselinic acid is a component of all detectable glycerolipids of developing carrot and coriander endosperm. Relative amounts of this fatty acid in lipid classes, however, were quite variable. As is the case with most unusual fatty acids, the highest levels of petroselinic acid were detected in TAG. In extracts of both carrot and coriander endosperm, petroselinic acid accounted for about 70 to $75 \mathrm{~mol} \%$ of the total fatty acid of TAG. Petroselinic acid was also the major fatty acid of DAG of both coriander and carrot endosperm, which is consistent with the primary tole of this lipid as a precursor of TAG in oil-accumulating seeds. In marked contrast, the major phospholipids PC, phosphatidylethanolamine (PE), and phosphatidic acid (PA), which together accounted for nearly $75 \mathrm{~mol} \%$ of the total polar lipids of carrot and coriander endosperm, contained about one-fourth to one-eighth as much petroselinic acid as TAG. For example, PC of carrot and coriander endosperm contained 15 and $20 \mathrm{~mol} \%$ petroselinic acid, respectively. In addition, petroselinic acid composed $<10 \mathrm{~mol} \%$ of PE of the endosperm of both species. In contrast to TAG, the major fatty acids of these polar lipids were oleic acid and its derivative $\alpha$-linoleic acid. Minor glycerolipids particularly phosphatidylinositol (PI) as well as the plastid galactolipids monogalactosyldiacylglycerol (MGDG) and digalactosyldiacylglycerol (DGDG) of carrot endosperm contained somewhat higher levels of petroselinic acid than did PC and PE. Still, relative amounts of petroselinic acid in these lipids were roughly one-half to one-third as much as in TAG.

In addition to petroselinic acid, small amounts $(\leq 1.5 \mathrm{~mol} \%)$ of other unusual fatty acids were

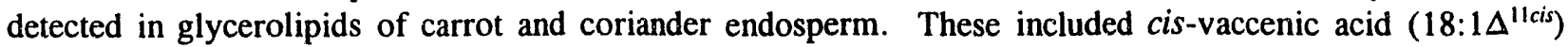
and three hexadecenoic acid (16:1) isomers. Mass spectral analyses of thiomethyl derivatives of hexadecenoic 
acid methyl esters indicated that these fatty acids were $\Delta^{4}, \Delta^{6}$, and $\Delta^{9}$ isomers (data not shown).

It was notable that PA levels in developing carrot and coriander endosperm were higher than might

be expected for a plant tissue. Slack et al. (1978), for example, reported that PA composed between 4 to $13 \mathrm{~mol} \%$ of the phospholipids of developing cotyledons of safflower, soybean, and linseed. In our analyses, PA accounted for approximately 16 and $24 \mathrm{~mol} \%$ of the polar lipids of the developing endosperm of coriander and carrot, respectively. It is difficult to attribute this PA to the degradation of PC and PE during or prior to lipid extraction. In order to inactivate potential lipolytic enzymes, endosperm was incubated in hot isopropanol before extraction of lipids (Kates and Eberhardt, 1957). Also, in radioisotope labelling experiments described below, PC was heavily labelled with $\left[1-{ }^{14} \mathrm{C}\right]$ acetate, but no significant breakdown of this lipid to PA was detected in either time-course or pulse-chase labelling experiments.

\section{Lipid Synthesis During the Development of Coriander Endosperm}

As described above, relative levels of petroselinic acid in phoshatidylcholine is more than one-fourth that in TAG. This difference might reflect temporal differences in PC and TAG synthesis. For example, the majority of PC may be synthesized during early stages of endosperm development prior to the rapid synthesis and accumulation of petroselinic acid in TAG. To examine this possibility, changes in absolute amounts of total fatty acid and petroselinic acid in PC and TAG were examined over a period of coriander endosperm development ranging from early to mid-maturity. Depending upon greenhouse conditions, a distinct endosperm (i.e., endosperm readily separable from pericarp) could be detected by approximately 12 to $16 \mathrm{DAF}$ and mid-maturity was reached by 18 to $22 \mathrm{DAF}$. For example, the fresh weight of coriander endosperm more than doubled between 16 and 20 DAF. During this period, TAG and PC were both actively synthesized as the fatty acid content of these lipids increased by more than six-fold and five-fold, respectively. Despite this, relative levels of petroselinic acid deposition into PC and TAG differed significantly. Of the change in fatty acid content of TAG, about $75 \%$ could be accounted for by increases in amounts of petrselinic acid [ $(\Delta$ nmol petroselinic acid $/ \Delta \mathrm{nmol}$ total fatty acid) $\times 100 \%]$. In contrast, changes in petroselinic acid content constituted only about $19 \%$ of the increases in fatty acids of PC of the developing coriander endosperm. These data therefore suggest that differences in relative amounts of petroselinic acid in PC and TAG are not the result of temporal differences in the synthesis of these lipids.

\section{[1 $\left.{ }^{14} \mathrm{C}\right]$ Acetate Time-Course Labelling of Carrot and Coriander Endosperm}

Analyses of fatty acid acid compositions of lipids and developmental changes in these compostions as described above give only an indication of the net products of a myriad of metabolic reactions. To gain a better understanding of how these fatty acid compostions arise, $\left[1{ }^{-14} \mathrm{C}\right]$ acetate labelling studies of carrot and coriander endosperm were performed. In time-course labelling of carrot endosperm, at least $85 \%$ of the label recovered in glycerolipids was detected in PC, TAG, DAG, and PE at time points through $1 \mathrm{~h}$. Of these lipids, PC and TAG were the most heavily labelled.

The most striking result was the rapid incorporation of high levels of $\left[1-{ }^{14} \mathrm{C}\right]$ acetate into $\mathrm{PC}$ as petroselinic acid. At time points through $30 \mathrm{~min}$, PC was the most heavily labelled glycerolipid. Of the radioactivity detected in PC during this period, nearly $85 \%$ was in the form of petroselinic acid, and even after $1 \mathrm{~h}, 75 \%$ of the label in PC was associated with this fatty acid. Relative to PC, the radiolabel appeared to initially enter TAG at a slower rate. However, by $1 \mathrm{~h}$, the highest amounts of radioactivity were detected in TAG. At this time point, TAG contained approximately 1.1 times as much ${ }^{14} \mathrm{C}$ as $\mathrm{PC}$. In addition, at least $90 \%$ of the radiolabel in T.AG was present as petroselinic acid throughout the $1 \mathrm{~h}$ labelling period. The incorporation of high amounts of $\left[1-{ }^{14} \mathrm{C}\right]$ acetate initially into PC followed by accumulation into TAG at later time points was suggestive of a precursor-product relationship between the fatty acids of PC and TAG. Such labelling kinetics, in fact, are similar to those previously described for the metabolism of oleic acid-derived $\mathrm{C}_{18}$ polyunsaturated fatty acids in seeds of soybean and linseed (Slack et al., 1978), in which PC was shown to be a precursor of TAG.

In addition to PC and TAG, $\left[1-{ }^{14} \mathrm{C}\right]$ acetate was incorporated into DAG and even the polar lipid PE primarily in the form of petroselinic acid. Throughout the $1 \mathrm{~h}$ time-course, $>80 \%$ of the label in these lipids was detected as petroselinic acid. 
Similar to carrot endosperm, in pulse-chase labelling experiments of coriander endosperm described below, $\left[1-{ }^{14} \mathrm{C}\right]$ acetate was also incorporated to high levels in $\mathrm{PC}$ as petroselinic acid during an initial $15 \mathrm{~min}$ "pulse" labelling period. In addition, in coriander endosperm that had been incubated in $\left[1-{ }^{14} \mathrm{C}\right]$ acetate for an extended period of time $(3.5 \mathrm{~h})$, PC persisted as a major labelled glycerolipid, accounting for nearly $30 \%$ of the total radioactivity recovered in the lipid extract. Of the label in PC, about $55 \%$ was detected as petroselinic acid. In comparison, $39 \%$ of the label in the total lipid extract was recovered as TAG, of which $90 \%$ was present in the form of petroselinic acid. DAG also contained significantly high levels of recovered label (19\% of the total label), and the relative proportion of radiolabelled petroselinic acid in this lipid (78\%) was roughly similar to that in the total extract. Although the total amount of label in PE was more than seven-fold less than that in PC, this polar lipid also contained high relative amounts of label in the form of petroselinic acid $(63 \%)$.

Suprisingly, in $\left[1-{ }^{14} \mathrm{C}\right]$ acetate labelling studies of both carrot and coriander endosperm describec above, the relative amounts of radiolabelled petroselinic acid incorporated into PC and PE were in large excess of that determined by fatty acid mass analyses. For example, in carrot endosperm, petroselinic acid composed $15 \mathrm{~mol} \%$ and $9 \mathrm{~mol} \%$ of the fatty acids of PC and PE, respectively. Yet, following $15 \mathrm{~min}$ incubation of this tissue in $\left[1-{ }^{14} \mathrm{C}\right]$ acetate, petroselinic acid accounted for $>80 \%$ of the radiolabelled fatty acids of these glycerolipids. In absolute terms, however, PC contained nearly seven thiAlso, the rate of fatty acid deposition into PC versus TAG as judged by net accumulation of fatty acid mass or by incorporation of $\left[1-{ }^{14} \mathrm{C}\right]$ acetate. As derived from, the net accumalation of fatty acid mass into TAG is about 35 times greater than that into PC in coriander endosperm. However, in the labelling studies described above, the accumulation of $\left[1-{ }^{14} \mathrm{C}\right]$ acetate into PC differed from that incorporated into TAG by less than a factor of 2 , even after incubation periods of $3.5 \mathrm{~h}$.

\section{DISCLAIMER}

This report was prepared as an account of work sponsored by an agency of the United States Government. Neither the United States Government nor any agency thereof, nor any of their employees, makes any warranty, express or implied, or assumes any legal liability or responsibility for the accuracy, completeness, or usefulness of any information, apparatus, product, or process disclosed, or represents that its use would not infringe privately owned rights. Reference herein to any specific commercial product, process, or service by trade name, trademark, manufacturer, or otherwise does not necessarily constitute or imply its endorsement, recommendation, or favoring by the United States Government or any agency thereof. The views and opinions of authors expressed herein do not necessarily state or reflect those of the United States Government or any agency thereof. 

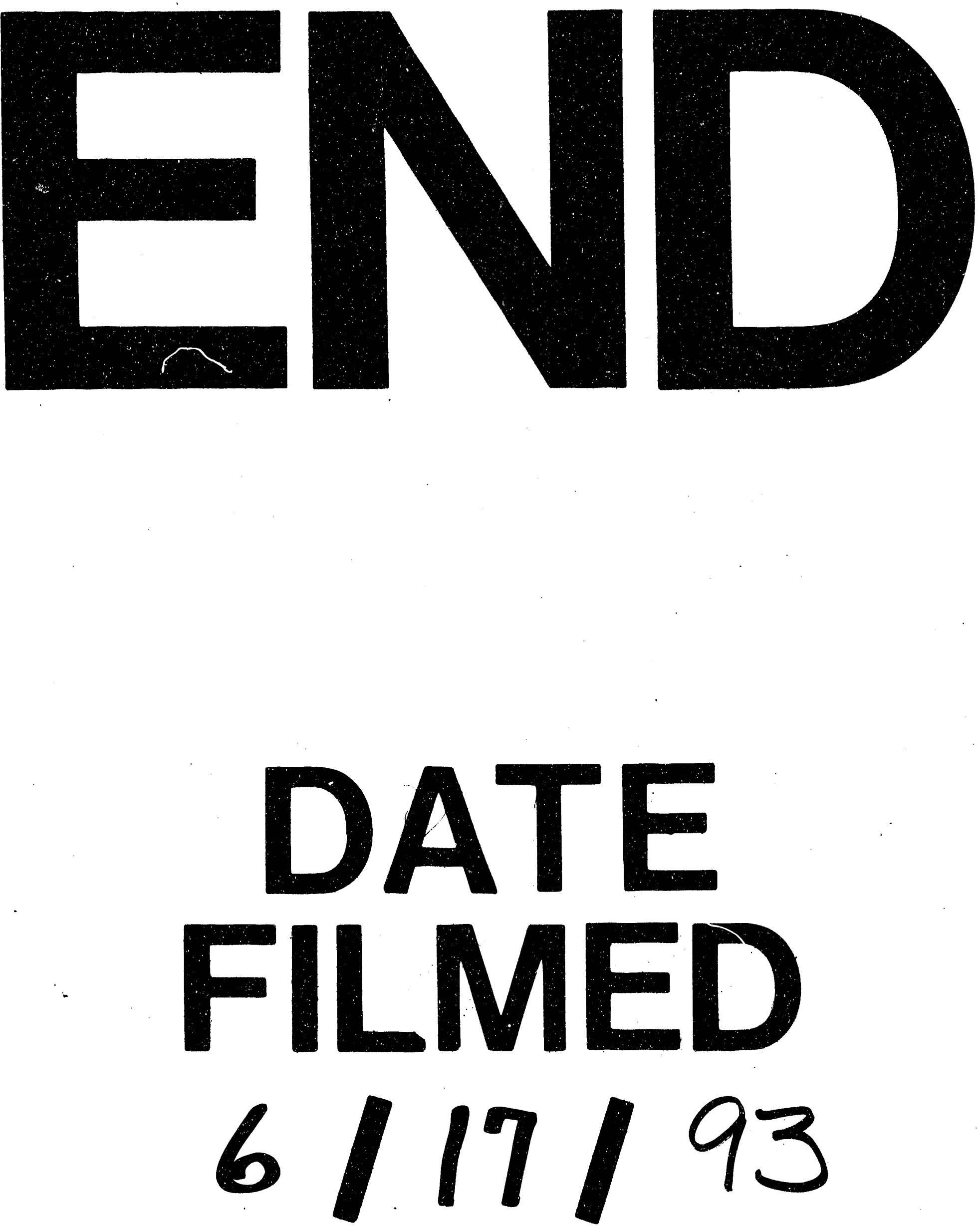
